\title{
Analysis of Retractions from Hospitals in Mainland China from 2017-2019
}

\section{Tianye Zhao}

Jilin University First Hospital

Tiancong Dai

Jilin University

\section{Zhijun Lun}

Jilin University First Hospital

\section{Yanli Gao ( $\nabla$ kjkgaoyanli@126.com )}

Jilin University First Hospital https://orcid.org/0000-0003-2204-1324

\section{Research}

Keywords: Hospitals, Scientific misconduct, Retraction of Publication as Topic, Plagiarism, Duplicate Publications as Topic

Posted Date: March 10th, 2020

DOI: https://doi.org/10.21203/rs.3.rs-16354/v1

License: (c) (i) This work is licensed under a Creative Commons Attribution 4.0 International License. Read Full License 


\section{Abstract}

Objective: The aim of this study is to analyze the features of retractions from hospitals in mainland China, and to discuss the causes of research misconduct by Chinese doctors.

Research Methods: PubMed, Web of Science, and Retraction Watch Database were searched to collect eligible records and to extract characteristics of the included entries, including publishers and Open Access status of the journals involved, ORCID, PubPeer comments before the retraction, whether there are authors from Grade A, Third-class hospitals, and whether there are response or requirements from authors.

Results: 521 retractions were included. Retractions were found primarily from authors of grade A, third-class hospitals, a limited regions, and published in journals with medium and high impact factor. The main reasons for retractions were Data Manipulation/Fabrication/Fraud(27.1\%), Error by Author(19.9\%), Plagiarism(16.7\%), Self-Plagiarism(9.1\%), Fake Peer Review(7.6\%) and Forged Authorship(6.3\%). Most of the retracted publications have neither ORCID nor PubPeer comments before their retraction.

Conclusion: This is the first report focus on the retractions from hospitals in mainland China. The large number of retractions from Chinese hospitals in recent years is worrying. The results suggests that some retractions are related to third-parties. Some features of retractions are centralized, and it is difficult to evaluate the role of ORCID and PubPeer in the retractions during this period.

\section{Introduction}

In 2017, Tumor Biology, a journal once indexed by SCIE, retracted 107 papers from mainland China in a single action(1). In following months, the Ministry of Science and Technology of the People's Republic of China investigated 521 authors and, found 486 of them responsible for their wrongdoing leading to the massive retractions(2). This incident raised widespread concerns about the quality of biomedical research articles from mainland China and marked the beginning of a series of investigations from publishers and scientific community. Retraction of publications is a troublesome issues, especially for biomedical journals, which may bring many negative consequences for authors, institutions, and journals, such as affecting the credibility of academic journals and causing lack of trust in medical science(3).

In 2018, the total number of science publications from China surpassed the United States for the first time(4), while a large number of retractions have come from hospitals in China. Many researchers have analyzed retractions in different disciplines, such as Al-Ghareeb(5) et al. in Nursing, Midwifery, Pantziarka(6) et al. in Oncology and Faggion(7) et al. in Dentistry. These studies have their value and may improve the fairness of peer reviews before and after publication, but few studies have focused on Chinese authors who are from hospitals. It is not a reasonable assumption that researchers who graduated from the same university and work in the same institution, just because they have entered different research fields, will differ in research misconduct, or there are differences in the integrity of researchers entering different fields. Therefore, we should pay attention to the role of authors and institutions in research misconduct and retractions, and especially analyze the characteristics of some specific groups among them. In this study, we reviewed 
retractions that occurred in hospitals in mainland China from 2017 to 2019, and focus on features that may explain the reasons for research misconduct among Chinese doctors.

\section{Material And Methods}

We collected and aggregate data from three databases, PubMed, Web of Science, and Retraction database(Version: 1.0.5.5) provided by Retraction Watch. The search in PubMed is. (Hospital[Affiliation]) AND (China[Affiliation]) Filters: Retraction of Publication, from 2017 to 2019. The search in Web of Science Core Collection is ADDRESS: (China) AND ADDRESS: (hosp) AND DOCUMENT TYPES: (Retracted Publication OR Retraction); Timespan: 2017-2019. Indexes: SCI-EXPANDED, SSCI. The search in Retraction database is the affiliation is "hospital AND China" and the timespan for retraction is from January 1, 2017 to December 31, 2019. Two reviewers independently filtered the search results to remove duplicate and irrelevant results.

Two reviewers independently classified the reasons for each article being retracted and if there is response or requirements from author(s), and the conflict between the two reviewers was determined by the supervisor. We categorize the reasons for retractions based on Retraction Watch Database User Guide(8). Specifically, the reasons can be divided into: (1)Self-Plagiarism: including duplication of article, data, image, and text. (2)Plagiarism: The publisher made a clear allegation of plagiarism. (3)Fake Peer Review. (4)Forged Authorship. (5)Data Manipulation/Fabrication/Fraud: The publisher made a clear allegation of fraud, or the reviewer is confident to think the scientific validity is impaired. (6)Ethical Violations. (7)Error by Author: The publisher claims that the authors wanted to retract their article or made vague allegations, and the reviewers were not confident that there was fraud. (8)Error by Publisher. (9)Cannot access retracted article notice. (10) No explanation is given. (11)Other. An article can be considered retracted for multiple reasons.

We get the journal's historical impact factor and open access status from Incites Journal Citation Reports provided by Clarivate Analytics and Journal's official website. Get the number of hospitals in November 2019 and the number of hospital visits from January to November 2019 by province from Center for Health Statistics and Information, National Health Commission of the People's Republic of China's official website( 9 , 10). For a very small number of retracted articles and retractions that are not available from the publisher's website, we try to obtain the historical version through SCI-HUB. Statistical analysis was completed in R 3.6.2.

\section{Results}

We obtained 1,979 records from PubMed, 2,006 records from Web of Science Core Collection, 547 records from Retraction database, and screened 521 articles for inclusion in the study. We describe the features of retracted articles in Table 1. Retraction styles vary, and most retractions give clear but limited information.

\section{Table 1 Description of the features of retracted articles.}




\begin{tabular}{|c|c|c|}
\hline Item & Number & Rate \\
\hline Total & 521 & - \\
\hline \multicolumn{3}{|l|}{ Retraction Time } \\
\hline 2017 & 161 & $30.9 \%$ \\
\hline 2018 & 149 & $28.6 \%$ \\
\hline 2019 & 211 & $40.5 \%$ \\
\hline \multicolumn{3}{|l|}{ Article Type } \\
\hline Research Article & 416 & $79.8 \%$ \\
\hline Clinical Study & 47 & $9.0 \%$ \\
\hline Review & 11 & $2.1 \%$ \\
\hline Meta-analysis & 35 & $6.7 \%$ \\
\hline Case Report & 9 & $1.7 \%$ \\
\hline Other & 3 & $0.6 \%$ \\
\hline \multicolumn{3}{|l|}{ Index } \\
\hline PubMed & 501 & $96.2 \%$ \\
\hline SCIE\&SSCI & 437 & $83.9 \%$ \\
\hline \multicolumn{3}{|c|}{ Published on Open Access Journal } \\
\hline Yes & 344 & $66.0 \%$ \\
\hline No & 177 & $34.0 \%$ \\
\hline \multicolumn{3}{|c|}{ With Author(s) from Grade A, Third-class hospitals } \\
\hline Yes & 499 & $95.8 \%$ \\
\hline No & 22 & $4.2 \%$ \\
\hline \multicolumn{3}{|l|}{ Author(s) with ORCID } \\
\hline Yes & 34 & $6.5 \%$ \\
\hline No & 487 & $93.5 \%$ \\
\hline \multicolumn{3}{|c|}{ Response or Requirements from Author(s) } \\
\hline Yes & 327 & $62.8 \%$ \\
\hline No & 49 & $9.4 \%$ \\
\hline Unmentioned/Inaccessible & 145 & $27.8 \%$ \\
\hline
\end{tabular}




\begin{tabular}{|lll|}
\hline Item & Number & Rate \\
\hline PubPeer Comment before Retraction & & \\
\hline Yes, with author response & 5 & $1.0 \%$ \\
\hline Yes, without author response & 33 & $6.3 \%$ \\
\hline No & 483 & $92.7 \%$ \\
\hline Retraction Reason & & \\
\hline Self-Plagiarism & 48 & $9.2 \%$ \\
\hline Plagiarism & 87 & $16.7 \%$ \\
\hline Fake Peer Review & 40 & $7.7 \%$ \\
\hline Forged Authorship & 33 & $6.3 \%$ \\
\hline Data Manipulation/Fabrication/Fraud & 143 & $27.4 \%$ \\
\hline Ethical Violations & 39 & $7.5 \%$ \\
\hline Error by Author & 102 & $19.6 \%$ \\
\hline Error by Publisher & 7 & $1.3 \%$ \\
\hline Cannot access retracted article notice & 17 & $3.3 \%$ \\
\hline No explanation is given & 49 & $9.4 \%$ \\
\hline Other & 19 & $3.6 \%$ \\
\hline
\end{tabular}

We found that 61 publishers involved, the top three is Springer Nature(101), Elsevier(83) and PLoS(59). More than a half retractions $(50.29 \%$ ) occur within two year after publication, with the longest lag time being 13 years. The range of impact factors for 518 cases that impact factor is available is $2.59[1.78,3.64]$, the maximum value is 23.24 .

In Fig. 2, the average number of outpatient visits per hospital in each province is displayed, as well as the number of retractions. Hospitals in mainland China generally have a large number of visits(average 106.4 thousand each hospital for 11 months), and retractions are concentrated in limited provinces. The spearman correlation analysis on them find significance(Spearman's rank correlation rho $=0.68, \mathrm{P}<0.001$ ). There is a positive association between average number of visits per hospital in provinces and number of retractions.

\section{Discussion}

In this study, we analyzed the retractions from hospitals in mainland China from 2017 to 2019, and paid attention to features of retractions and retracted publications like whether there were PubPeer comments 
issued before the retraction, whether the author used ORCID, whether there are authors came from grade $A$, third-class hospitals (not just third-class hospital), and whether there are response or requirements from authors, which were rarely reported before. As a small number of retractions were not correctly labeled in the database, we acknowledge that we may not have collected all retractions that should be included.

Many hospitals in China have a complex form as academic institutions, and this is important in two ways. One is that doctors receive different administration in scientific research and clinical work, and the other is that the scientific research platforms available to different doctors sometimes lack comparability. In addition, there may be research departments are run by full-time researchers, which is more common to major hospitals. Large hospitals, especially the grade A, third-class hospitals, need doctors to contribute their research results, which is one of the contents of evaluating the performance of hospital management(11). However, at the same time, hospitals may not be able to provide adequate support for doctors in scientific research, especially for hospitals that are not teaching hospitals in medical schools. Doctors need to do scientific research activities in laboratories in other institutions or commission others to perform experiments. A considerable number of Chinese doctors want to learn and publish Meta-analysis, which has formed a tendency, but hospitals possibly does not provide sufficient database accesses. These may cause difficulties in maintaining original records, difficulties in monitoring the research process by the corresponding author, and motivating doctors to find other solutions. Hospitals have a positive attitude towards acknowledging their articles, but have a negative attitude towards accountability for research misconduct(12). Doctors in China need to publish papers in academic journals for promotion and economic benefits. Young doctors face fierce competitions, especially in Third-class hospitals, and their pressures to publish in internationally indexed journals were described as "Perish or Publish"(13). Chinese doctors are under tremendous clinical work pressure, most of them work more than forty hours a week(14). Finally, some factors cause these busy doctors to choose research misconduct, since we found that provinces with larger average hospital visits have more retractions. But this is not to say that they cannot conduct scientific research. Some doctors and graduate students can get involved in research for a period of time without participating in clinical work. In addition, some doctors conduct scientific research at night and on rest days. Nonetheless, our results suggest that the more busy doctors are, the more retractions happened is not true. Although in the research process, there may be unfair access to academic resources, in recent years academic journals have been fairly fair to recognize academically valuable articles from Chinese doctors. Impact factors, journal divisions, these quantitative indicators provide a predictable channel for doctors to get rid of their disadvantaged position. The reasonable standards of international journals on alternative medicine also limit the impact of nonmedical causes. Chinese doctors, especially those in large hospitals, have conflicting attitudes towards publishing papers. Different doctors have different ideas, and they have different ideas in different periods, depending on whether they can win in this competition. But even those at a disadvantage in this race may not be willing to accept another set of qualitative and vague rules. As a result, doctors invest a lot of effort in writing articles, even if they will occasionally like the post that calls for cancellation of quantitative evaluation.

A common view is that academic journals should explain the reasons for retractions in more detail and in a more standardized way $(15,16)$. In this study, sometimes we need some help from PubPeer comments to figure out the reason for retractions, because the retraction notice is not clear. However, journals need the 
cooperation of authors to obtain sufficient evidence. In the case of insufficient evidence, journals often choose vaguely word retractions. In an intriguing example, the journal has announced that the experimental methods described by the authors were not able to get the results they reported, but they have not made up their minds to retract that article, and just advised readers to "interpret these results with caution"(17). Some retractions indicate that the author refused to respond to publisher inquiries, nevertheless, we observed that in 37 cases the editors still accused and retracted the articles. We suggest that hospitals should list refusals to respond to editor's inquiries and failure to find original experimental results as reasons for punishment, not just retractions.

Although the reasons for many retractions are not clearly given, we can still speculate on some reasons based on our research. Some authors seem unfamiliar with academic norms and do not have any sense of academic integrity. For example, a group of authors published four Meta-analyses that were highly similar except for the substance in 2014, and two of them were identified as self-plagiarized(18-20). The details mentioned in another retraction may explain this phenomenon. The authors clarified that they referred to templates to write their article, which from a training course on how to write Meta-analyses(21). Some retractions from hospitals in mainland China due to self-plagiarism, plagiarism, fake peer review, forged authorship and data manipulation/fabrication/fraud is confirmed to involve third parties, which has been mentioned by several retractions(22-24). In other words, we guess that many Chinese doctors are not able to complete some fraud independently. These third parties usually charge high fees, and may use aggressive violations to help clients publish articles. In a case of forged authors and plagiarism, a third party got an unpublished manuscript from an agency for language editing, and used that to write an article for their clients, which brought tricky trouble for the real authors(25). In another case, it may be due to a lack of communication that a third party published the manuscript in another journal after the author had already published it(26). In other cases, the results were purchased by the author from a third party, and they can not ensure the reliability of their articles(27). For example, the editor stated in a retraction that the authors' contributions to the work were "unclear" in their perspective(28). A third party provided the same results to two research teams, and the articles of both teams solved the same research questions and reported the same data, results, and conclusions(29). Recent investigations have suggested the presence of paper mills(30). They use manuscript templates to produce a lot of similar articles, regular and similar results pictures, and submit these articles to various journals for his client. Some Chinese doctors may be involved, but this is a new discovery that received the attention of the public only in 2020(31), and it is not yet reflected in the results we have collected in these three years. It is worth noting that there are tens of retractions due to ethical issues occurred, which mentioned similar reasons, and similarly worrying matters behind(32).

We counted several external features of retracted articles and retractions, and found that the retractions were concentrated in grade A, third-class hospitals, a limited regions, and medium and high-impact journals. Explanations of these phenomena require qualitative interviews to be published. There are not many low impact factor articles, probably because the benefits are too small compared to the risk of research misconduct. The post-publish peer review website PubPeer provides an effective platform for senior scientists to point out that they have found research misconduct. However, it is difficult to evaluate the role of PubPeer in these retractions from 2017 to 2019, as most articles(92.7\%) do not have any comments posted before they were retracted. Only a small number of comments were answered by the author, and the author's 
response was generally decent. We found ORCID information in a few articles(6.5\%), but most did not show any public information. Although we did not see the current role of ORCID in holding researchers responsible for their retractions, we can still be optimistic. We suggest ORCID to consider that the retraction records are showed and visible to the trusted parties or everyone by default on ORCID homepage, which can accurately increase the adverse consequences of research misconduct. As more and more researchers use ORCIDs, publishers can force authors to submit their ORCIDs and associated retraction records. Even if the hospital subscribes to a small academic database, it can review applicants' ORCID accounts to check if they have retracted manuscripts when hiring and promoting doctors. Hospitals should record the e-mail addresses and ORCIDs of senior doctors, as using one-time e-mail addresses or ORCID submissions is suspect.

The phenomenon that a large number of retractions from Chinese hospitals in recent years is really worrying. Hospitals and medical libraries should take some measures to reduce the occurrences of research misconduct. In addition to the measure that seems to solve the problem fundamentally, but it is actually impossible to be promoted by individual hospitals: reform the doctor evaluation system, there are some shortterm and mild measures that can be taken. In a prospective study on perceptions of Chinese Biomedical researchers towards research misconduct among Chinese biomedical researchers, more than $60 \%$ of participants thought that the punishment for research misconduct from authority and institutions was not severe enough(12). In fact, few hospitals in China have detailed rules and enforce them transparently, putting researchers with retracted publications at a disadvantage to their long-term interests, such as relegation, be limited to access to academic resources, and no promotion for several years(33). In 2019, the National Press and Publication Administration released the first industry standard against misconduct entitled Academic Publishing Specification-Definition of Academic Misconduct for Journals (CY/T174-2019)(34). This document provides a reference for hospital to design rules. In addition, it can be asserted that most of the retractions did not actually have widespread social impact in mainland China, regardless of whether the reasons were sufficiently interesting. Medical library should educate doctors, particularly in the early stages of their career, which will help them cultivate an ethical research system(35). The training includes introduction to academic publishing, peer review, and unique identifiers for researchers, as well as instruction on the rational use of software, because if doctors can use the tool software themselves, they may not buy thirdparty services. Libraries should also consider inviting journal editors, which can be Chinese journals or English journals, to introduce doctors to effective methods of communication with editors. The hospital's library should consider collecting the raw data and experimental records submitted by the doctor, so that when the doctor refuses to answer the editor's inquiry, or jumps to another hospital and cannot be contacted, the hospital can have a way to reduce the research misconduct discovered to the institution's reputation loss. In our position, we do not want researchers from hospitals in mainland China to face any discrimination, but we hope that the improvement of the status quo begins with valuing academic integrity, improving the quality of research, and stopping the unreasonable pushing of doctors to publish papers.

\section{Conclusion}

This is the first report focus on the retractions from hospitals in mainland China. The number of retractions from hospitals in mainland China from 2017 to 2019 is worrying large. Most retractions are related to research misconduct, some of which have been confirmed or are likely to be related to third parties. Some 
features of retractions are centralized, and it is difficult to evaluate the role of ORCID and PubPeer in the retractions during this period.

\section{Abbreviations}

ORCID: Open Researcher and Contributor ID

hosp: hospital

SCIE: Science Citation Index Expanded.

SSCI: Social Sciences Citation Index.

\section{Declarations}

\section{Ethics approval and consent to participate}

Not applicable.

\section{Consent for publication}

Not applicable.

\section{Availability of data and materials}

The datasets used and analyzed during the current study are available from the corresponding author on reasonable request.

\section{Competing interests}

The authors declare that they have no competing interests.

\section{Funding}

None.

\section{Authors' contributions}

Zhao and Dai reviewed the data. Zhao was a major contributor in writing the manuscript. Lun and Gao supervised the project. All authors read and approved the final manuscript. Lun and Gao contributed equally to this manuscript.

\section{Acknowledgements}

Thanks to Retraction Watch for providing and maintaining their database, Zhefan Shi for helping in collecting the data, and TigerBB8 for suggesting to improve this study. 


\section{References}

1. Stigbrand T. Retraction Note to multiple articles in Tumor Biology. Tumour Biol. 2017.

2. the Ministry of Science and Technology of the People's Republic of China. [Press conference on the investigation and handling of centralized retractions of academic journals held in Beijing]: the Ministry of Science and Technology of the People's Republic of China,. 2017. Available from: http://www.most.gov.cn/kjbgz/201707/t20170727_134289.htm. Accessed 21 February 2020.

3. Campos-Varela I, Villaverde-Castaneda R, Ruano-Ravina A. Retraction of publications: a study of biomedical journals retracting publications based on impact factor and journal category. Gac Sanit. 2019.

4. Tollefson J. China declared world's largest producer of scientific articles. Nature. 2018;553(7689):390.

5. Al-Ghareeb A, Hillel S, McKenna L, Cleary M, Visentin D, Jones M, et al. Retraction of publications in nursing and midwifery research: A systematic review. Int J Nurs Stud. 2018;81:8-13.

6. Pantziarka P, Meheus L. Journal retractions in oncology: a bibliometric study. Future Oncol. 2019;15(31):3597-608.

7. Faggion CM, Jr., Ware RS, Bakas N, Wasiak J. An analysis of retractions of dental publications. J Dent. 2018;79:19-23.

8. Retraction Watch. Retraction Watch Database User Guide Appendix B: Reasons. Available from: https://retractionwatch.com/retraction-watch-database-user-guide/retraction-watch-database-user-guideappendix-b-reasons/. Accessed 21 February 2020.

9. Center for Health Statistics and Information. [National Medical Services in January-November 2019]: National Health Commission of the People's Republic of China,. 2020. Available from: http://www.nhc.gov.cn/mohwsbwstjxxzx/s7967/202001/55e9bcc9829e41278ea29d15e2ad10c1.shtml. Accessed 21 February 2020.

10. Center for Health Statistics and Information. [Number of national health institutions at the end of November 2019]: National Health Commission of the People's Republic of China,. 2020. Available from: http://www.nhc.gov.cn/mohwsbwstjxxzx/s7967/202001/d73a47fbda0e4ea4bbb8d20387992871.shtml. Accessed 21 February 2020.

11. Wanqiang $X$, Jianghua $L$, Wenbin W. Research and prospect of hospital scientific research performance management. Chinese Journal of Hospital Administration. 2019;35(7):599-602.

12. Liao QJ, Zhang YY, Fan YC, Zheng MH, Bai Y, Eslick GD, et al. Perceptions of Chinese Biomedical Researchers Towards Academic Misconduct: A Comparison Between 2015 and 2010. Sci Eng Ethics. 2018;24(2):629-45.

13. Tian M, Su Y, Ru X. Perish or Publish in China: Pressures on Young Chinese Scholars to Publish in Internationally Indexed Journals. Publications. 2016;4(2):9.

14. Zhang C, Liu Y. The salary of physicians in Chinese public tertiary hospitals: a national cross-sectional and follow-up study. BMC Health Serv Res. 2018;18(1):661-.

15. Hesselmann F, Graf V, Schmidt M, Reinhart M. The visibility of scientific misconduct: A review of the literature on retracted journal articles. Curr Sociol. 2017;65(6):814-45. 
16. Nair S, Yean C, Yoo J, Leff J, Delphin E, Adams DC. Reasons for article retraction in anesthesiology: a comprehensive analysis. Can J Anaesth. 2020;67(1):57-63.

17. Li L, Qin J, Fu T, Shen J. Expression of Concern: Fisetin rescues retinal functions by suppressing inflammatory response in a DBA/2J mouse model of glaucoma. Doc Ophthalmol. 2019;139(3):169.

18. Yang X, Lv S, Zhou X, Liu Y, Li D, Shi R, et al. Retraction Note to: The Clinical Implications of Transforming Growth Factor Beta in Pathological Grade and Prognosis of Glioma Patients: A Meta-Analysis. Mol Neurobiol. 2017;54(3):2378.

19. Lv S, Dai C, Liu Y, Shi R, Tang Z, Han M, et al. Retraction Note to: The Impact of Survivin on Prognosis and Clinicopathology of Glioma Patients: A Systematic Meta-Analysis. Mol Neurobiol. 2017;54(3):2376.

20. Yang X, Lv S, Liu Y, Li D, Shi R, Tang Z, et al. Retraction Note to: The Clinical Utility of Matrix Metalloproteinase 9 in Evaluating Pathological Grade and Prognosis of Glioma Patients: A MetaAnalysis. Mol Neurobiol. 2017;54(3):2377.

21. BioMed Research I. Retracted: Influence of Two Common Polymorphisms in the EPHX1 Gene on Warfarin Maintenance Dosage: A Meta-Analysis. Biomed Res Int. 2019;2019:6910869-.

22. Guo X, Sun W, Huang L, Wu L, Hou Y, Qin L, et al. Retracted: Effect of Cinnamaldehyde on Glucose Metabolism and Vessel Function. Med Sci Monit. 2018;24:4572.

23. Retraction note: Investigation of the hub genes and related mechanism in ovarian cancer via bioinformatics analysis. J Ovarian Res. 2015;8(1):15.

24. Retraction note: Risk miRNA screening of ovarian cancer based on miRNA functional synergistic network. J Ovarian Res. 2015;8(1):17.

25. Retraction: Nucleophagy in Human Disease: Beyond the Physiological Role. [Tohoku J. Exp. Med., 2018, 244 (1), 75-81. doi: 10.1620/tjem.244.75. Review.]. Tohoku J Exp Med. 2018;244(2):175.

26. Hu M, Liu B. Retraction notice to: Resveratrol attenuates lipopolysaccharide-induced dysfunction of blood-brain barrier in endothelial cells via AMPK activation. Korean J Physiol Pharmacol. 2017;21(2):277.

27. Editors PO. Retraction: Human Phosphatidylethanolamine-Binding Protein 4 Promoted the Radioresistance of Human Rectal Cancer by Activating Akt in an ROS-Dependent Way. PLoS One. 2019;14(1):e0210896.

28. Editors PO. Retraction: rs10499194 polymorphism in the tumor necrosis factor-alpha inducible protein 3 (TNFAIP3) gene is associated with type-1 autoimmune hepatitis risk in Chinese Han population. PLoS One. 2018;13(3):e0195181.

29. Editors PO. Retraction: Overexpression of Rad51 Predicts Poor Prognosis in Colorectal Cancer: Our Experience with 54 Patients. PLoS One. 2018;13(11):e0206398.

30. Byrne JA, Christopher J. Digital magic, or the dark arts of the 21 st century-how can journals and peer reviewers detect manuscripts and publications from paper mills? FEBS Letters.n/a(n/a).

31. Clyde S. The full-service paper mill and its Chinese customers: LEONID SCHNEIDER; 2020. Available from: https://forbetterscience.com/2020/01/24/the-full-service-paper-mill-and-its-chinese-customers/. Accessed 21 February 2020. 
32. Shazi L, Abbas Z. Ethical dilemmas related to living donor liver transplantation in Asia. Ir J Med Sci. 2019;188(4):1185-9.

33. Wang L, Liu Z. Keeping a clean research environment: Addressing research misconduct and improving scientific integrity in China. Cancer Lett. 2019;464:1-4.

34. Zhu J. New Official Documents of China Addressing Academic Misconduct. Sci Eng Ethics. 2019.

35. Cyranoski D. China introduces 'social' punishments for scientific misconduct. Nature. 2018;564(7736):312.

\section{Figures}

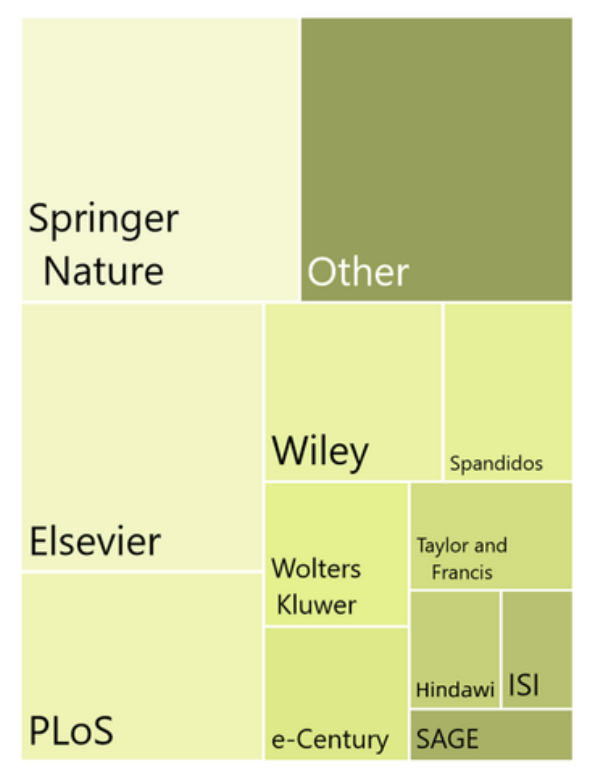

Publishers

(a)

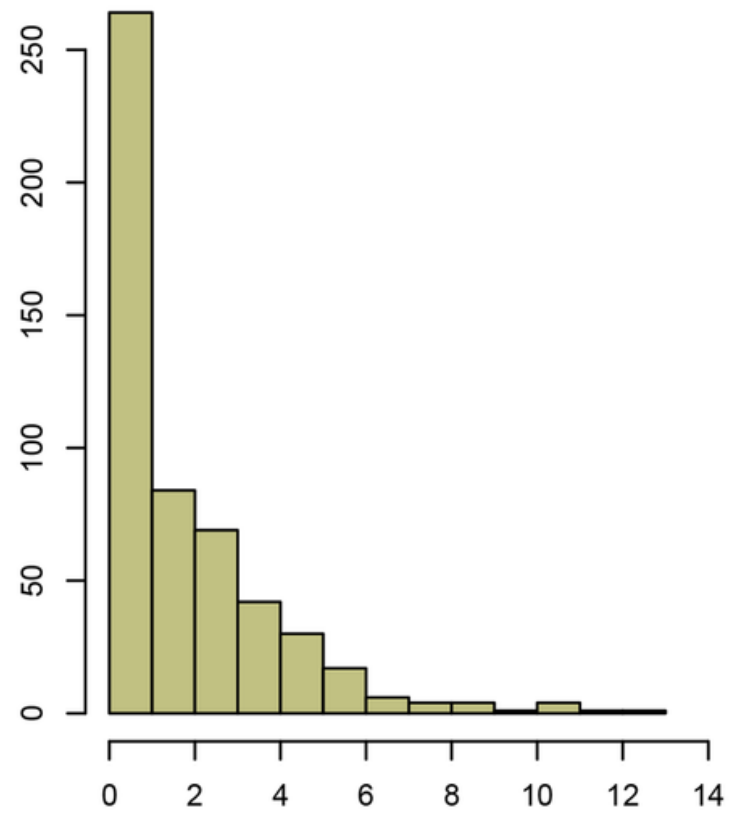

Years from publication to retraction

(b)

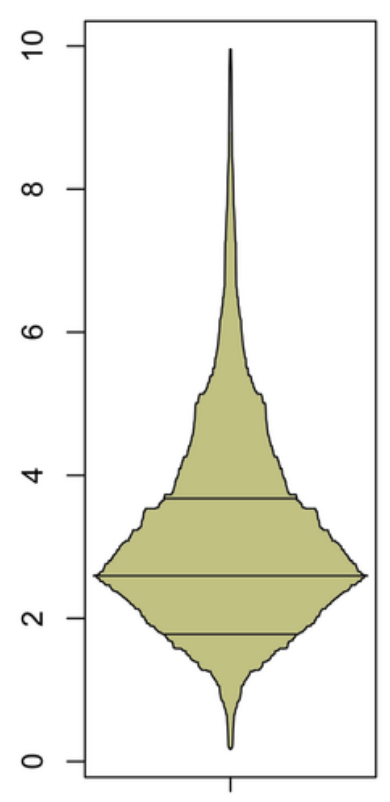

Impact Factor

(c)

\section{Figure 1}

(a) Treemap for publishers involved. (b) Histogram for years from publication to retraction(rounded down).

(c) Box-Percentile Plot for impact factor of journals. The data are asymmetrically centered around the median. The impact factor is one year prior to publication, as it reflects the information the author was facing at the time of submission, and if not accessible, the most recent data available. Data greater than 10 are not shown in the figure. 


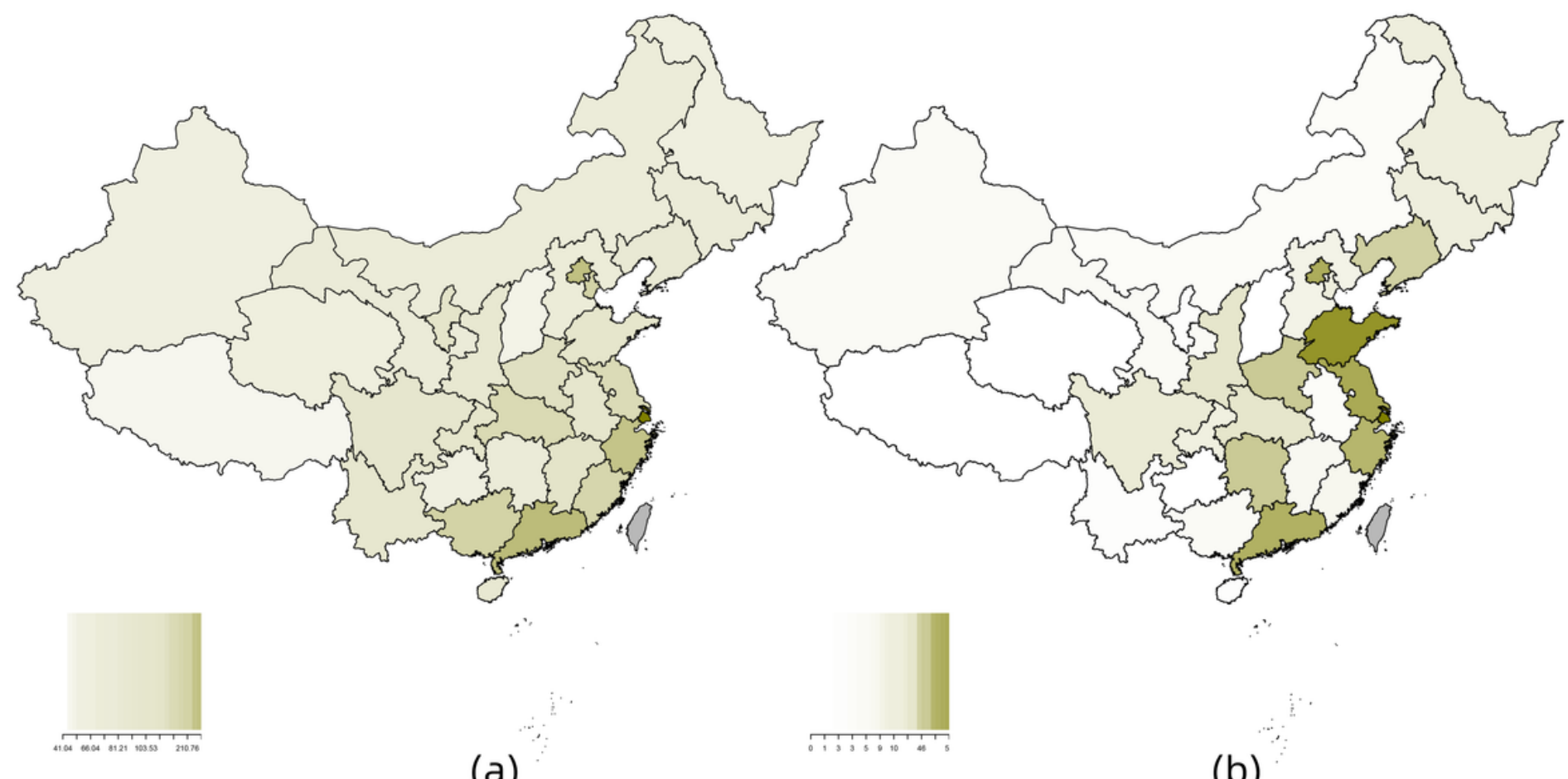

(a)

(b)

\section{Figure 2}

(a) Average number(thousand) of visits per hospital during January to November 2019 in each province of mainland China. (b) Retraction during 2017-2019 in each provinces in mainland China. In both maps, the darker the color, the greater the number. Note: The designations employed and the presentation of the material on this map do not imply the expression of any opinion whatsoever on the part of Research Square concerning the legal status of any country, territory, city or area or of its authorities, or concerning the delimitation of its frontiers or boundaries. This map has been provided by the authors. 